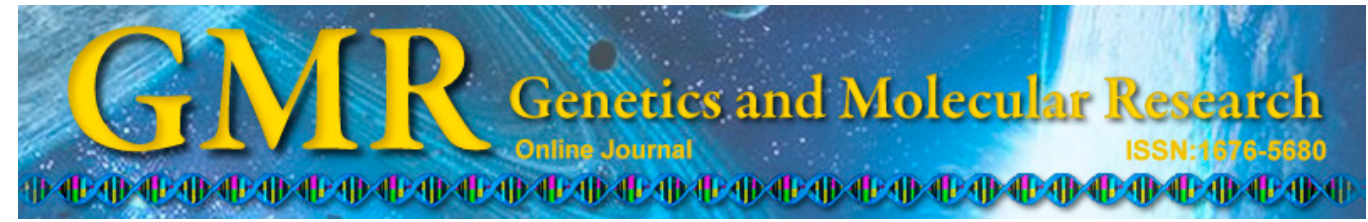

\title{
Correlation analysis of ultrasonic characteristics, pathological type, and molecular markers of thyroid nodules
}

\author{
J.J. Su${ }^{1}$, L.Z. Hui ${ }^{2}$, C.J. Xi ${ }^{2}$ and G.Q. Su ${ }^{2}$ \\ ${ }^{1}$ Department of Ultrasound Diagnosis, The First Affiliated Hospital, \\ Xiamen University, Xiamen, China \\ ${ }^{2}$ Department of General Surgery, The First Affiliated Hospital, \\ Xiamen University, Xiamen, China \\ Corresponding author: G.Q. Su \\ E-mail: guoqiangsu@126.com
}

Genet. Mol. Res. 14 (1): 9-20 (2015)

Received September 24, 2014

Accepted December 19, 2014

Published January 15, 2015

DOI http://dx.doi.org/10.4238/2015.January.15.2

\begin{abstract}
The present study was conducted to analyze the correlation between ultrasonic characteristics, pathological type, and molecular markers of thyroid-tumor-related genes as well as to evaluate the diagnosis and prognosis of thyroid nodules. The acoustic characteristics of 130 thyroid specimens were detected. Pathological sectioning and immunohistochemical detection were performed to determine the correlation between tumor gene expression and ultrasonic characteristics. Ultrasonic testing revealed that malignant nodules were normally accompanied by lymph nodes. Expression of the human telomerase reverse transcriptase, Ki67, vascular endothelial growth factor, Ret, and P53 genes exhibited statistically significant differences in malignant, benign, and normal tissues. The performance of thyroid malignant nodules showed different degrees of correlation with the expression of the human telomerase reverse transcriptase, Ki67, VEGF, Ret, and P53 genes. Color Doppler ultrasound is highly sensitive for thyroid nodules and is therefore
\end{abstract}


effective for identifying thyroid nodules and early diagnosis of thyroid cancer. Color Doppler ultrasound can identify benign or malignant thyroid nodules based on 5 characteristic indicators. Tumor pathology and gene expression are associated with the sonographic features of thyroid cancer. Therefore, determining the pathological basis of ultrasonography would facilitate prognostic assessments of thyroid cancer.

Key words: Differential diagnosis; Histopathology; Thyroid nodule; Tumor gene; Ultrasound

\section{INTRODUCTION}

Thyroid nodule is a common disorder of the endocrine system. Most thyroid nodules are benign tumors or indicative of hyperplasia, whereas approximately $5 \%$ of these nodules are malignant (Davies and Welch, 2006). The incidence of thyroid cancer has gradually increased over the past 20 years, accounting for the highest number of head and neck cancers. Therefore, accurate diagnosis and proper treatment of thyroid nodules, particularly the early detection and timely treatment of thyroid cancer, are very important.

The American Thyroid Association (Cooper et al., 2006) defined thyroid nodules as "thyroid disorders which could be discriminated from the surrounding thyroid parenchyma, using palpation or ultrasound methods" (Morris et al., 2008). This definition indicates the importance of ultrasound methods in identifying thyroid nodules. The ATA has further reiterated that ultrasound shows high diagnostic importance in thyroid cancer. They also defined low level echo, obscure boundary, aspect ratio $>1$, microcalcification, and rich blood as acoustic characteristics that can be used to diagnose malignant nodules. Thus, color ultrasound, particularly high-resolution ultrasound imaging technology, not only has advantages, such as non-invasiveness, real-time monitoring, low cost, and ease of operation, but can also clearly reveal the number, size, and shape of the thyroid lesion, therefore making it the first option for discriminating and diagnosing thyroid nodules (Miyakawa, 2012).

However, the development of benign thyroid nodules into cancer involves multiple genes and is a multi-step process, during which multi-genetic mutations and changes in related cytokines occur (Endo et al., 2008; Fagin and Mitsiades, 2008). The existence of various types of thyroid tumors increases the difficulty of ultrasonic diagnosis; thus, misdiagnosis still occurs in ultrasonic testing.

Previous studies have shown that ultrasound-guided fine-needle aspiration biopsy and the detection of certain molecular markers of thyroid cancer can facilitate the diagnosis and evaluation of prognosis. Moreover, facilitating clinical outcome assessment for developing individualized treatment programs is necessary (Lee et al., 2012; Rossi et al., 2012; Nikiforov et al., 2013).

The relationships between the acoustic characteristics of thyroid nodules and cancer-related genes [including the telomerase, Ret, P53, and vascular endothelial growth factor (VEGF) genes] (Cantara et al., 2012; Messina et al., 2012; Borrello et al., 2013), as well as their corresponding pathological features were analyzed in this study. We applied color Doppler ultrasound in the identification and prognostic evaluation of thyroid nodules. 


\section{MATERIAL AND METHODS}

\section{Subjects}

A total of 120 nodular specimens with surgical ablation that were pathologically diagnosed as benign or malignant thyroid tumors were collected from 110 cases from January 2009 to September 2012 in our hospital. Of the 120 specimens, 30 were benign thyroid nodules and 90 were thyroid cancer nodules (another 10 para-nodule normal thyroid tissues were utilized as controls). The subjects included 34 males and 86 females aged 32-69 years with a mean age of $46 \pm 3.5$ years. Among the 30 cases of benign thyroid nodules, 17 were nodular goiters and 13 were thyroid adenomas. Among the malignant cases, 75 were papillary carcinomas, 11 were follicular carcinomas, 1 was a medullary carcinoma, 3 were undifferentiated carcinomas, and 27 were accompanied by lymph node metastasis. According to TNM staging of tumor (the 6th edition of Cancer Staging Manual published in 2002, by Greene et al.), 41 cases were in stage I, 31 cases were in stage II, 12 cases were in stage III, and 6 cases were in stage IV; among patients older than 45 years, 4 exhibited bone metastases and two exhibited pulmonary metastases. No patients had undergone preoperative treatment with integral imaging data and clear imaging. This study was conducted in accordance with the Declaration of Helsinki and with approval from the Ethics Committee of Xiamen University. Written informed consent was obtained from all participants.

\section{Ultrasonic inspection}

The LOGIQ7 color ultrasound (GE Healthcare; Little Chalfont, UK) was utilized in this study. The probe frequency was 7.5-10 MHz. Preliminary examination was performed with the preset thyroid parameters. The gain, depth, and focus area were adjusted according to the specific circumstances of lesions to achieve the best display effect. The patient was placed in the supine position without a pillow for thyroid scanning. When cervical lymph nodes had been scanned, the patient was placed in the supine position with his or her head moved from side to side for scanning. Two-dimensional (2D) and color Doppler ultrasound scans were performed first on the thyroid and then on the 2 sides of the neck and lymph nodes above the bilateral collarbone.

\section{Thyroid ultrasound scanning}

For 2D ultrasound scanning, the following sonographic features were recorded: number, size, location, shape, boundaries, acoustic halo, echo intensity, echo uniformity, and microcalcifications of the thyroid nodule.

For color Doppler flow imaging, we referred to Adler et al. (1990) blood flow richness grading method.

For lymph node scanning on the 2 sides of the neck and collarbone, we referred to Antonelli et al. (2003) criteria.

\section{Pathological examination}

The location, size, number, texture, and slice features of the nodules were observed. Experienced pathologists and sonographers reviewed the pathological diagnosis and diag- 
nosed the cases as thyroid tumors. Lesions from hematoxylin and eosin staining with ultrasound recording were retained for future review.

\section{Immunohistochemical detection}

Paraffined tissue sections were dewaxed, dewatered, and then rinsed with water. Tissue antigens were prepared according to the requirements of each antibody (e.g., citric acid with high pressure for $1.5 \mathrm{~min}$ ) and washed 3 times with phosphate-buffered saline (PBS) for 3-5 min. Next, the PBS solution was removed, and a drop of $50 \mu \mathrm{L} \mathrm{3 \%}$ hydrogen peroxide was added to each slice followed by incubation at room temperature for $10 \mathrm{~min}$ to block endogenous peroxidase activity. Then the slices were washed with PBS for 3 times, 3 min for each time. A $50-\mu \mathrm{L}$ drop non-immune goat serum (10\%) was added to each slice followed by incubation at room temperature for $10 \mathrm{~min}$. The residual solution was directly discarded.

A total of $50 \mu \mathrm{L}$ primary antibody was added to each slice followed by incubation at room temperature for $60 \mathrm{~min}$. The slices were then washed 3 times with PBS for 3-5 min. The PBS solution was removed and $50 \mu \mathrm{L}$ MaxVision (KIT-5910 MaxVision HRP-polymer anti-rabbit/ mouse IHC Kit; Fuzhou Maixin Biotechnology Development, Co., Ltd.; Guangdong, China) was added to each slice. The slices were incubated at room temperature for $15 \mathrm{~min}$ and then washed 3 times with PBS for 3 min each time. The PBS solution was removed and $100 \mu \mathrm{L}$ freshly prepared 3,3'-diaminobenzidine chromogenic solution was added to each slice. Slices were observed under a microscope after $5 \mathrm{~min}$, rinsed with water, restained with hematoxylin, rinsed with PBS or water to return to a blue color, and dehydrated with gradient alcohol. Next, the slices were hyalinized with xylene and then cemented with neutral gum. Human telomerase reverse transcriptase (hTERT) rabbit anti-human monoclonal antibody, Ki67 mouse anti-human monoclonal antibody, VEGF rabbit anti-human monoclonal antibody, and anti-P53 mouse anti-human monoclonal antibody were purchased from Fuzhou Maixin Biotechnology Development Co., Ltd. Ret rabbit antihuman single monoclonal antibody was purchased from Abcam (HK) Ltd., Hong Kong, China.

\section{Determination of immunohistochemical results}

The resulting $34-\mu \mathrm{m}$ sections of thyroid lesions were fixed in $10 \%$ formaldehyde solution, embedded in paraffin, and stained with hematoxylin and eosin for immunohistochemical staining (S-P immunohistochemical method) and 3,3'-diaminobenzidine coloration according to the immunohistochemistry kit instructions. The following criteria were employed (US Image Pro Plus computer image analysis system; Media Cybernetics; Rockville, MD, USA). First, brown indicated a positive result. P53 protein was localized in the cytoplasm and the nucleus. hTERT and Ki67 proteins were localized in the nucleus, while VEGF and Ret proteins were localized to the cytoplasm and cell membrane. Ten non-overlapping and representative highly magnified fields $(200 \mathrm{X})$ were selected from each specimen. Images were examined using a high-resolution color medical image analysis system. Average gray and surface density values were then calculated. Second, the average gray value for each case was calculated according to the average gray value of each field. hTERT expression intensity was considered to be the average gray value. The average gray value was inversely proportional to intracellular contents and immunohistochemical staining intensity. The surface density value was calculated using the same method and was found to be positively proportional to intracellular substances and immunohistochemical expression intensity. 


\section{Statistical analysis}

Data were analyzed using the SPSS 13.0 statistical software (SPSS, Inc.; Chicago, IL, USA). $\mathrm{P}<0.05$ was considered to be statistically significant. Measured data are reported as the means \pm standard deviation. Count data were compared using the chi-square test, and multivariate factorial comparison was performed for the analysis of variance.

\section{RESULTS}

\section{Pathological results}

The pathological results showed that among the 30 cases of benign thyroid nodules, 17 were nodular goiters and 13 were thyroid adenomas. Among the malignant cases, 75 were papillary carcinomas, 11 were follicular carcinomas, 1 was medullary carcinomas, and 3 were undifferentiated carcinomas.

\section{2-D ultrasound performance of thyroid nodules}

Most benign thyroid nodules exhibited a regular shape, which was oval or circular, and had relatively smooth borders. Internal echo was cystic and solid cystic. The calcified lesion was coarse, typically arranged in a circle, or was arc-shaped. The aspect ratio was $<1$, and was generally observed without cervical lymphadenopathy (Figure 1A and B).

$\mathbf{A}$

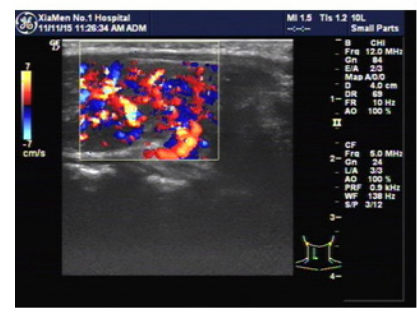

D

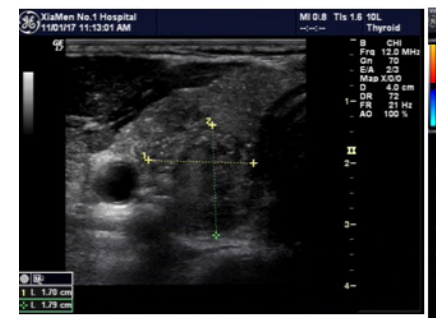

B

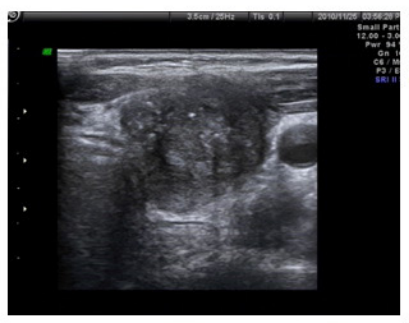

$\mathbf{E}$

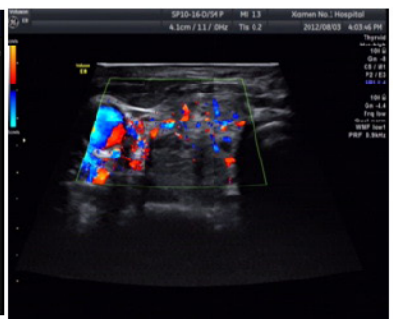

$\mathbf{C}$

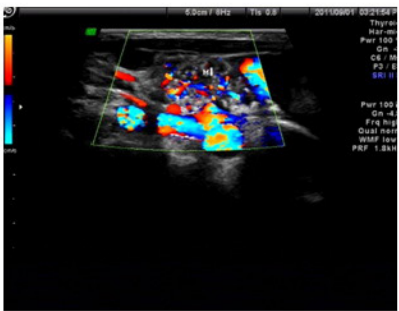

$\mathbf{F}$

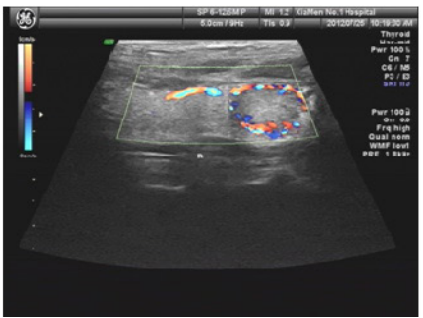

Figure 1. Ultrasonic Characteristics of benign, malignant thyroid nodules, and metastasis lymph nodes. A. Nodular goiter: multiple cystic nodules, less clear boundary. B. Thyroid adenoma: hypoechoic, clear boundary, peripheral blood flow. C. Papillary thyroid carcinoma: kernel cystic nodule, rich blood. D. Papillary thyroid carcinoma: hypoechoic nodules, vague boundary, irregular shape, with sand-like calcification. E. Papillary thyroid carcinoma: vague boundary, irregular shape with sand-like calcification, the aspect ratio was greater than one. F. Thyroid cancer with lymph node calcification, rich blood. 
Malignant nodules were generally irregular and had rough boundaries. Internal echo was mostly hypoechoic solid nodules, and the calcified lesions often appeared as sand granules with an irregular arrangement. The aspect ratio was $\geq 1$ and was occasionally accompanied by cervical lymphadenopathy (Figure $1 \mathrm{C}$ and E; Table 1).

\begin{tabular}{|c|c|c|c|c|c|c|c|c|c|c|c|}
\hline \multirow[t]{2}{*}{ Groups } & \multicolumn{2}{|c|}{ Boundary } & \multicolumn{2}{|c|}{ Internal echo } & \multicolumn{2}{|c|}{ Calcification } & \multicolumn{2}{|c|}{$\begin{array}{l}\text { Ratio of longitudinal } \\
\text { and transverse diameter }\end{array}$} & \multicolumn{2}{|c|}{ Blood flow } & \multirow[t]{2}{*}{ Summary } \\
\hline & Clear & Obscure & Low & Others & Microcalcification & Others & $<1$ & $\geq 1$ & Level 3 & Others & \\
\hline Benign & 30 & 0 & 1 & 29 & 0 & 30 & 28 & 2 & 0 & 30 & 30 \\
\hline Malignant & 51 & 39 & 77 & 13 & 66 & 24 & 55 & 35 & 79 & 11 & 90 \\
\hline Summary & 81 & 39 & 78 & 42 & 66 & 54 & 83 & 37 & 79 & 41 & 120 \\
\hline$\kappa^{2}$ & \multicolumn{2}{|c|}{29.57} & \multicolumn{2}{|c|}{66.86} & \multirow{2}{*}{\multicolumn{2}{|c|}{$\begin{array}{l}48.89 \\
<0001\end{array}$}} & \multicolumn{2}{|c|}{10.954} & \multicolumn{2}{|c|}{77.07} & \\
\hline $\mathrm{P}$ & \multicolumn{2}{|c|}{$<0.001$} & \multicolumn{2}{|c|}{$<0.001$} & & $<0.001$ & \multicolumn{2}{|c|}{$<0.001$} & \multicolumn{2}{|c|}{$<0.001$} & \\
\hline
\end{tabular}

\section{Color Doppler performance of benign thyroid nodules and thyroid cancer}

The blood flow signals of the thyroid nodules in the color Doppler showed that among the 30 cases of benign nodules, 22 were Class I and 5 were Class II. Among the 90 cases of thyroid cancer, 3 were Class I, 8 were Class II, and 79 were Class III (Table 1; Figure 1C).

\section{Ultrasonic performance of thyroid cancer lymph node metastasis}

Among the 120 cases of thyroid nodules, benign nodules did not exhibit lymph node metastasis. Among the 90 cases of malignant nodules, only 27 occurred with lymph node metastasis and exhibited the following ultrasonic occurrences: 1) 7 cases had strong echo of intralymph nodes, uneven distribution, and sand-like calcification; 2) 4 cases exhibited kernel intralymph rich blood flow signals, which may have been due to liquefaction or cystic degeneration and disordered blood direction with fast and stubby arterial blood vessels; 3) 10 cases showed irregular thickening of the lymph nodes, and the cortex and medulla were reduced or their structure had disappeared; 4) 6 cases exhibited swollen lymph nodes with a round shape and a reduced axial ratio (Figure $1 \mathrm{~F}$ ).

\section{Comparative analysis of preoperative ultrasonic acoustic features and postoperative pathological diagnosis}

Significant differences were observed in the occurrence of 5 characteristics, including unclear boundary, low echo, microcalcification, aspect ratio $(\geq 1)$, and blood flow (Level 3$)$ in benign and malignant nodules. The sensitivities of these 5 indicators, which were treated as separate indicators for the diagnosis of malignant thyroid nodules, were 56.7, 85.6, 73.3, 38.9, and $87.8 \%$, respectively. In the diagnosis of benign thyroid nodules, the sensitivities were 100 , $96.6,100,93.3$, and $100 \%$, respectively (Table 2 ).

\section{Relationship between oncogene expression and ultrasound imaging features}

No expression of the tumor genes hTERT, Ki67, VEGF, Ret, and P53 was observed 
in benign thyroid nodules and the normal thyroid group. One case of benign thyroid adenoma presented low expression levels of hTERT and Ret; staining intensity was weak and the particles were only dot-scattered. Thus, the pathologists identified the 2 genes as unexpressed. The gene expression of malignant nodules was significantly higher than those in the normal and benign groups, with values of $82.2,77.8,85.6,90$, and $85.6 \%$, respectively; the difference was significant, whereas the gene expression of the benign and normal groups exhibited no significant difference (Table 3, Figure 2).

Table 2. Comparison of ultrasonic feature and postoperative pathological diagnosis of 120 cases.

\begin{tabular}{|c|c|c|c|c|c|c|}
\hline \multirow[t]{2}{*}{ Ultrasonic feature } & \multicolumn{2}{|c|}{ Benign group } & \multirow[t]{2}{*}{ Sensitivity } & \multicolumn{2}{|c|}{ Malignant group } & \multirow[t]{2}{*}{ Sensitivity } \\
\hline & + & - & & + & - & \\
\hline Unclear boundary & 0 & 30 & $100 \%$ & 51 & 39 & $56.7 \%$ \\
\hline Low echo & 1 & 29 & $96.6 \%$ & 77 & 13 & $85.6 \%$ \\
\hline Microcalcification & 0 & 30 & $100 \%$ & 66 & 24 & $73.3 \%$ \\
\hline Ratio of longitudinal and transverse demeter & 2 & 28 & $93.3 \%$ & 35 & 55 & $38.9 \%$ \\
\hline Blood flow & 0 & 30 & $100 \%$ & 79 & 11 & $87.8 \%$ \\
\hline
\end{tabular}

Ratio of longitudinal and transverse Demeter $\geq 1$, and the blood flow referred to Level 3 blood flow.

Table 3. Tumor gene expressions of 130 cases of benign, malignant thyroid nodules, and normal group.

\begin{tabular}{|c|c|c|c|c|c|c|c|c|c|c|c|}
\hline \multirow[t]{2}{*}{ Groups } & \multirow[t]{2}{*}{ Cases } & \multicolumn{2}{|c|}{ hTERT } & \multicolumn{2}{|c|}{ Ki67 } & \multicolumn{2}{|c|}{ VEGF } & \multicolumn{2}{|c|}{ Ret } & \multicolumn{2}{|c|}{ P53 } \\
\hline & & + & - & + & - & + & - & + & - & + & - \\
\hline Benign & 30 & 0 & 30 & 0 & 30 & 0 & 30 & 0 & 30 & 0 & 30 \\
\hline Normal & 10 & 0 & 10 & 0 & 10 & 0 & 10 & 0 & 10 & 0 & 10 \\
\hline Malignant & 90 & 74 & 16 & 70 & 20 & 77 & 13 & 81 & 9 & 77 & 13 \\
\hline Summary & & 74 & 56 & 70 & 60 & 77 & 53 & 81 & 49 & 77 & 53 \\
\hline$\kappa^{2}$ & & \multicolumn{2}{|c|}{76.349} & \multicolumn{2}{|c|}{67.407} & \multicolumn{2}{|c|}{83.941} & \multicolumn{2}{|c|}{95.510} & \multicolumn{2}{|c|}{83.941} \\
\hline $\mathrm{P}$ & & \multicolumn{2}{|c|}{$<0.001$} & \multicolumn{2}{|c|}{$<0.001$} & \multicolumn{2}{|c|}{$<0.001$} & \multicolumn{2}{|c|}{$<0.001$} & \multicolumn{2}{|c|}{$<0.001$} \\
\hline
\end{tabular}

$\mathbf{A}$

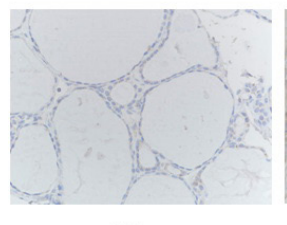

F

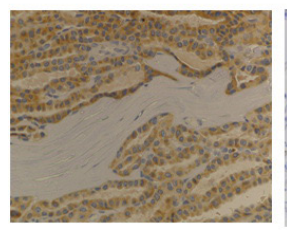

B

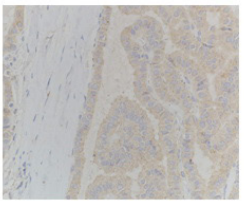

G

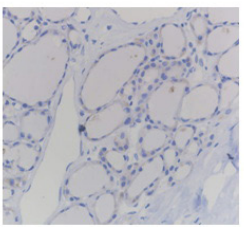

C

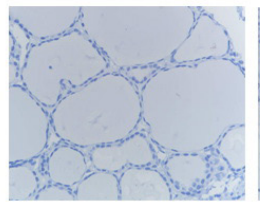

$\mathbf{H}$

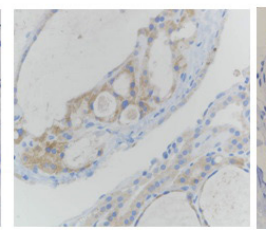

D

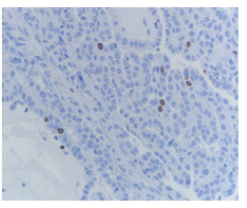

I

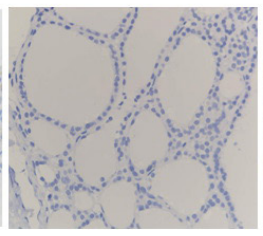

$\mathbf{E}$

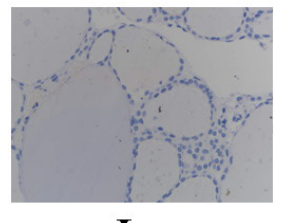

$\mathbf{J}$

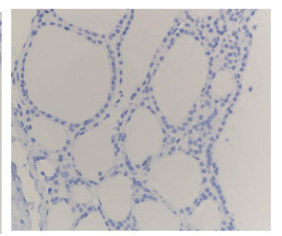

Figure 2. Expression of oncogene expression (200X) in benign, malignant thyroid nodules. A. hTERT expression in benign nodules. B. hTERT expression in malignant nodules. C. Ki67 expression in benign nodules. D. Ki67 expression in malignant nodules. E. VEGF expression in benign nodules. F. VEGF expression in malignant nodules. G. Ret expression in benign nodules. H. Ret expression in malignant nodules. I. P53 expression in benign nodules. J. P53 expression in malignant nodules. 
Positive expression levels of hTERT in low-level echo, microcalcifications, Class III blood, aspect ratio, and lymph node metastasis were higher than values in the control group ( $\mathrm{P}$ $<0.01$ ). However, the unclear boundary was not significantly different (Table 4).

\begin{tabular}{|c|c|c|c|c|c|c|c|c|c|c|c|c|}
\hline \multirow[t]{2}{*}{ hTERT } & \multicolumn{2}{|c|}{ Boundary } & \multicolumn{2}{|c|}{ Internal echo } & \multicolumn{2}{|c|}{ Calcification } & \multicolumn{2}{|c|}{$\begin{array}{l}\text { Ratio of longitudinal } \\
\text { and transverse diameter }\end{array}$} & \multicolumn{2}{|c|}{ Blood flow } & \multicolumn{2}{|c|}{ Lymph nodes } \\
\hline & Clear & $\overline{\text { Obscure }}$ & Low & Others & Microcalcification & Others & $<1$ & $\geq 1$ & Level 3 & Others & + & - \\
\hline Positive group & 48 & 26 & 71 & 3 & 60 & 14 & 31 & 43 & 70 & 4 & 25 & 49 \\
\hline Negative group & 33 & 13 & 7 & 39 & 6 & 40 & 6 & 40 & 9 & 37 & 2 & 44 \\
\hline Summary & 81 & 39 & 78 & 42 & 66 & 54 & 37 & 83 & 79 & 41 & 27 & 93 \\
\hline$\kappa^{2}$ & \multicolumn{2}{|c|}{0.611} & \multicolumn{2}{|c|}{81.261} & \multicolumn{2}{|l|}{53.056} & \multicolumn{2}{|c|}{11.070} & \multicolumn{2}{|c|}{70.994} & \multicolumn{2}{|c|}{14.095} \\
\hline$P$ & \multicolumn{2}{|c|}{0.434} & \multicolumn{2}{|c|}{$<0.001$} & \multicolumn{2}{|l|}{$<0.001$} & \multicolumn{2}{|c|}{$<0.001$} & \multicolumn{2}{|c|}{$<0.001$} & \multicolumn{2}{|c|}{$<0.001$} \\
\hline
\end{tabular}

The positive expression levels of Ki67 in low-level echo, unclear boundary, microcalcifications, Class III blood, and aspect ratio were significantly higher than those in the control group $(\mathrm{P}<0.01)$. Lymph node metastasis did not exhibit any significant difference with respect to Ki67 expression (Table 5).

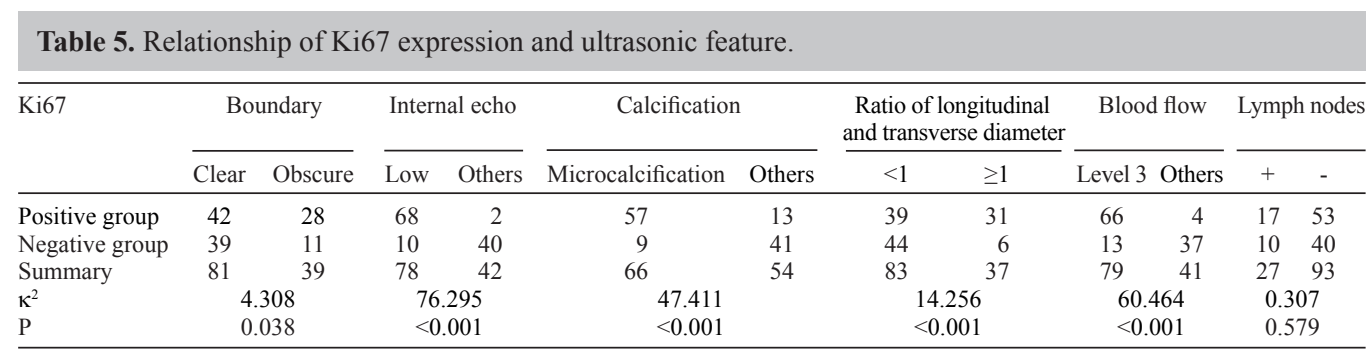

Positive expression levels of VEGF in unclear boundary, low-level echo, microcalcifications, Class III blood, aspect ratio, and lymph node metastasis were significantly higher than those in the control group $(\mathrm{P}<0.01)$ (Table 6).

\begin{tabular}{|c|c|c|c|c|c|c|c|c|c|c|c|c|}
\hline \multirow[t]{2}{*}{ VEGF } & \multicolumn{2}{|c|}{ Boundary } & \multicolumn{2}{|c|}{ Internal echo } & \multicolumn{2}{|l|}{ Calcification } & \multicolumn{2}{|c|}{$\begin{array}{c}\text { Ratio of longitudinal } \\
\text { and transverse diameter }\end{array}$} & \multicolumn{2}{|c|}{ Blood flow } & \multicolumn{2}{|c|}{ Lymph nodes } \\
\hline & Clear & Obscure & Low & Others & Microcalcification & Others & $<1$ & $\geq 1$ & Level 3 & Others & + & - \\
\hline Positive group & 46 & 31 & 72 & 5 & 64 & 13 & 44 & 33 & 72 & 5 & 24 & 53 \\
\hline Negative group & 35 & 8 & 6 & 37 & 2 & 41 & 39 & 4 & 7 & 36 & 3 & 40 \\
\hline Summary & 81 & 39 & 78 & 42 & 66 & 54 & 83 & 37 & 79 & 41 & 27 & 93 \\
\hline$\kappa^{2}$ & \multicolumn{2}{|c|}{5.898} & \multicolumn{2}{|c|}{76.756} & \multicolumn{2}{|l|}{68.638} & \multicolumn{2}{|c|}{14.567} & \multicolumn{2}{|c|}{73.160} & \multicolumn{2}{|c|}{9.261} \\
\hline $\mathrm{P}$ & \multicolumn{2}{|c|}{0.015} & \multicolumn{2}{|c|}{$<0.001$} & \multicolumn{2}{|l|}{$<0.001$} & \multicolumn{2}{|c|}{$<0.001$} & \multicolumn{2}{|c|}{$<0.001$} & \multicolumn{2}{|c|}{0.002} \\
\hline
\end{tabular}

Positive expression levels of RET in unclear boundary, low-level echo, microcalcifications, Class III blood, aspect ratio, and lymph node metastasis were significantly higher than those in the control group $(\mathrm{P}<0.01)$ (Table 7). 


\begin{tabular}{|c|c|c|c|c|c|c|c|c|c|c|c|c|}
\hline \multirow[t]{2}{*}{ Ret } & \multicolumn{2}{|c|}{ Boundary } & \multicolumn{2}{|c|}{ Internal echo } & \multicolumn{2}{|c|}{ Calcification } & \multicolumn{2}{|c|}{$\begin{array}{l}\text { Ratio of longitudinal } \\
\text { and transverse diameter }\end{array}$} & \multicolumn{2}{|c|}{ Blood flow } & \multicolumn{2}{|c|}{ Lymph nodes } \\
\hline & Clear & Obscure & Low & Others & Microcalcification & Others & $<1$ & $\geq 1$ & Level 3 & Others & + & - \\
\hline Positive group & 46 & 35 & 69 & 12 & 62 & 19 & 48 & 33 & 75 & 6 & 26 & 55 \\
\hline Negative group & 35 & 4 & 9 & 30 & 4 & 35 & 35 & 4 & 4 & 35 & 1 & 38 \\
\hline Summary & 81 & 39 & 78 & 42 & 66 & 54 & 83 & 37 & 79 & 41 & 27 & 93 \\
\hline$\kappa^{2}$ & \multicolumn{2}{|c|}{13.031} & \multicolumn{2}{|c|}{44.636} & \multicolumn{2}{|l|}{46.736} & \multicolumn{2}{|c|}{11.471} & \multicolumn{2}{|c|}{79.342} & \multicolumn{2}{|c|}{13.169} \\
\hline $\mathrm{P}$ & \multicolumn{2}{|c|}{$<0.001$} & \multicolumn{2}{|c|}{$<0.001$} & \multicolumn{2}{|l|}{$<0.001$} & \multicolumn{2}{|c|}{0.001} & \multicolumn{2}{|c|}{$<0.001$} & \multicolumn{2}{|c|}{$<0.001$} \\
\hline
\end{tabular}

Positive expression levels of P53 in unclear boundary, low-level echo, microcalcifications, Class III blood, aspect ratio, and lymph node metastasis were significantly higher than those in the control group $(\mathrm{P}<0.01)$ (Table 8).

\begin{tabular}{|c|c|c|c|c|c|c|c|c|c|c|c|c|}
\hline \multirow[t]{2}{*}{ P53 } & \multicolumn{2}{|c|}{ Boundary } & \multicolumn{2}{|c|}{ Internal echo } & \multicolumn{2}{|l|}{ Calcification } & \multicolumn{2}{|c|}{$\begin{array}{l}\text { Ratio of longitudinal } \\
\text { and transverse diameter }\end{array}$} & \multicolumn{2}{|c|}{ Blood flow } & \multicolumn{2}{|c|}{ Lymph nodes } \\
\hline & Clear & Obscure & Low & Others & Microcalcification & Others & $<1$ & $\geq 1$ & Level 3 & Others & + & - \\
\hline Positive group & 42 & 35 & 68 & 9 & 57 & 20 & 43 & 34 & 68 & 9 & 24 & 53 \\
\hline Negative group & 39 & 4 & 10 & 33 & 9 & 34 & 40 & 3 & 11 & 32 & 3 & 40 \\
\hline Summary & 81 & 39 & 78 & 42 & 66 & 54 & 83 & 37 & 79 & 41 & 27 & 93 \\
\hline$\kappa^{2}$ & \multicolumn{2}{|c|}{16.438} & \multicolumn{2}{|c|}{51.330} & \multicolumn{2}{|l|}{31.42} & \multicolumn{2}{|c|}{17.884} & \multicolumn{2}{|c|}{48.271} & \multicolumn{2}{|c|}{9.261} \\
\hline $\mathrm{P}$ & \multicolumn{2}{|c|}{$<0.001$} & \multicolumn{2}{|c|}{$<0.001$} & \multicolumn{2}{|l|}{$<0.001$} & \multicolumn{2}{|c|}{0.001} & \multicolumn{2}{|c|}{$<0.001$} & \multicolumn{2}{|c|}{0.002} \\
\hline
\end{tabular}

\section{DISCUSSION}

Thyroid nodule is a common neck disease that generally affects approximately $3-7 \%$ of the population as detected by palpation; up to $20-76 \%$ of cases are detected through highresolution ultrasound (Gharib et al., 2010), among which 5-15\% are malignant thyroid nodules (thyroid cancer) (Cooper et al., 2009). The treatment and prognosis of benign and malignant thyroid nodules are quite different. Therefore, the assessment of thyroid nodules should focus on distinguishing between benign and malignant nodules. In this study, high-resolution ultrasound was employed to detect thyroid nodules and the expression of cancer-related genes was measured to provide a basis for the diagnosis and prognosis of thyroid nodules. The following acoustic characteristics of benign thyroid nodules were observed: regular shape with smooth borders, the internal echo was mainly a mixture of cystic and cystic solid echoes, coarse calcifications, aspect ratio $<1$, and absence of cervical lymphadenopathy. Malignant nodules did not exhibit these acoustic characteristics. A number of acoustic indicators can be combined to further improve diagnostic sensitivity. Similar findings have been reported previously. Papini et al. (2002) reported that combining any 2 characteristics such as solid low-level echo, microcalcification, vague boundary, and blood flow can be used to detect $87 \%$ of thyroid cancer cases; nodules requiring needle biopsy accounted for $30 \%$. Cappelli et al. (2005) combined the use of any 2 of aspect ratio $>1$, microcalcification, vague boundary, and blood flow and detected $83 \%$ of thyroid cancer cases, among which only $15 \%$ required fine-needle aspiration biopsy. 
The following results were obtained from further correlation analysis of ultrasound characteristics and the pathology of thyroid nodules. Microcalcifications showed $73.3 \%$ diagnostic sensitivity for thyroid cancers (66/90), with 100\% specificity. Microcalcifications refer to calcifications less than $1 \mathrm{~mm}$ in diameter as observed on ultrasound images; these calcifications reflect the pathological sand body. Klinck and Winship (1959) reviewed 2153 patients with benign thyroid disease and found that only 1 patient had a sand body. Another study reported that microcalcification is specific to thyroid cancer, and that the specificity could reach $80-85.5 \%$ (Frates et al., 2006). Thus, the presence of a sand body is important for the diagnosis of thyroid cancer.

In the present study, 22 cases from the malignant group exhibited coarse calcification, which may have been related to the malignancy of nodular goiter and thyroid adenoma. No significant calcification was observed in the 2 cases of papillary carcinoma, which could mean that the disease was still in the early stage and calcifications had not yet appeared.

Several studies reported that ultrasonic low-level echo of thyroid cancer accounts for $71 \%$ of cases. Thyroid cancer cells are large and overlap with minimal interstitial space, resulting in a strong reflection interface and a hypoechoic appearance. In the present study, the incidence of hypoechoic lesions in the malignant group was $85.6 \%$ (77/90). Only 1 case exhibited a hypoechoic appearance in the benign group, indicating that internal low echo is important in diagnosing thyroid cancer. Thirteen cases of thyroid cancer exhibited a cystic or cystic solid echo, which may have been related to the secondary lesions and degenerative changes in thyroid nodules, such as cystic degeneration or bleeding. One study suggested that papillary thyroid carcinoma and undifferentiated carcinoma more than $1 \mathrm{~cm}$ in diameter could cause bleeding and cystic degeneration (Senchenkov and Staren, 2004). Papillary thyroid carcinoma has $10-20 \%$ occurrence of cystic degeneration.

Thyroid nodules are irregularly shaped. The boundary is vague and the typical sonographic appearance of thyroid cancer shows no acoustic halo. A typical thyroid cancer biopsy has a star-shaped appearance; the boundary is unclear, and the boundary between the cancer cells and the surrounding tissues is also unclear. Thus, invasion into surrounding tissues can be observed. The boundary was clear in the malignant nodules in the present study, leading to the following observations: 1 ) some tumors were discovered early; the tumor was small and significant invasion was not yet observable; 2) some tumors did not exhibit a high degree of malignancy; 3) fibrous stromal hyperplasia formed fibrous pseudocapsules, retaining the clear boundary between the lesion and the surrounding thyroid tissues; 4) the rich level and distribution of blood was associated with the pathological type of thyroid nodules. The richness of blood flow was mostly 0 and of Class I in benign nodules, revealing that the internal and peripheral nodules had no blood flow or had point-like and minimal blood flow (blood flow distribution was mostly of type I). In malignant nodules, the abundance of blood was attributed to Class II and Class III, reflecting richer internal and peripheral blood flow signals (blood flow distribution was mostly of type II). Although some benign nodules show rich internal blood flow, the internal flow signals of malignant nodules are messy, irregular, and different from those in benign nodules. However, several studies have shown that blood flow distribution cannot be used as a basis for the differential diagnosis of benign and malignant nodules (Desser and Kamaya, 2008). The flow index is less reliable for differentiating between benign and malignant thyroid lesions. In this study, patients with thyroid carcinoma comprised 79 cases of Class III blood flow (79/90, 87.7\%). Very few Class III cases were observed in benign 
nodules, indicating that blood richness levels of thyroid nodules can be used as an important reference in the identification of benign and malignant thyroid nodules.

The aspect ratio of thyroid nodules is important for predicting thyroid cancer, particularly in thyroid microcarcinoma diagnosis. Previous study (Qi et al., 2012) suggest that this result may be related to the growth of thyroid cancer, in which the very small thyroid cancers often overcome the oppression of capsular tension and exhibit lateral growth; ultrasound images show full nodules with a transversely oval shape, and the aspect ratio is $\geq 1$ and perpendicular to the organs. Common benign nodules show a longitudinal oval shape. In this study, the nodule aspect ratio was $\geq 1$. A sensitivity of $38.9 \%$ was exhibited in the diagnosis of thyroid cancer, and a low index was observed, which may be related to the low incidence or detection rate of small cancers.

Analysis of tumor molecular markers and thyroid pathology revealed no expression of the tumor genes $h T E R T$, Ki67, VEGF, Ret, and P53 in benign thyroid nodules. One case of benign thyroid adenoma exhibited very weak expression of hTERT and Ret; however, as the staining intensity was weak and the particles were only dot-scattered, the pathologists identified the case as unexpressed. Gene expression in malignant nodule tumors was significantly higher than those in the normal and benign groups with expression levels of 82.2, 77.8, 85.6, 90 , and $85.6 \%$, respectively. Nikiforov et al. (2013) combined fine-needle aspiration biopsy and RET testing and compared the results with those from fine-needle aspiration cell biopsy alone. The sensitivity and specificity of the preoperative diagnosis of benign and malignant thyroid nodules improved, suggesting that the integrated use of different means of detection increases the accuracy of the diagnosis of malignant thyroid nodules. Gene expression levels showed varying degrees of relevance with the sonographic features of thyroid nodules, further illustrating the importance of tumor marker detection in the diagnosis, prognosis, and turnover of tumors.

\section{REFERENCES}

Adler DD, Carson PL, Rubin JM and Quinn-Reid D (1990). Doppler ultrasound color flow imaging in the study of breast cancer: preliminary findings. Ultrasound Med. Biol. 16: 553-559.

Antonelli A, Miccoli P, Fallahi P, Grosso M, et al. (2003). Role of neck ultrasonography in the follow-up of children operated on for thyroid papillary cancer. Thyroid 13: 479-484.

Borrello MG, Ardini E, Locati LD, Greco A, et al. (2013). RET inhibition: implications in cancer therapy. Expert. Opin. Ther. Targets 17: 403-419.

Cantara S, Pisu M, Frau DV, Caria P, et al. (2012). Telomere abnormalities and chromosome fragility in patients affected by familial papillary thyroid cancer. J. Clin. Endocrinol. Metab. 97: E1327-E1331.

Cappelli C, Pirola I, Cumetti D, Micheletti L, et al. (2005). Is the anteroposterior and transverse diameter ratio of nonpalpable thyroid nodules a sonographic criteria for recommending fine-needle aspiration cytology? Clin. Endocrinol. 63: 689-693.

Cooper DS, Doherty GM, Haugen BR, Kloos RT, et al. (2006). Management guidelines for patients with thyroid nodules and differentiated thyroid cancer. Thyroid 16: 199-142.

Cooper DS, Doherty GM, Haugen BR, Kloos RT, et al. (2009). Revised American Thyroid Association management guidelines for patients with thyroid nodules and differentiated thyroid cancer. Thyroid 19: 1167-1214.

Davies L and Welch HG (2006). Increasing incidence of thyroid cancer in the United States, 1973-2002. JAMA 295: 2164-2167.

Desser TS and Kamaya A (2008). Ultrasound of thyroid nodules. Neuroimaging Clin. N. Am. 18: 463-78.

Endo T, Ohta K and Kobayashi T (2008). Expression and function of Cbfa-1/Runx2 in thyroid papillary carcinoma cells. J. Clin. Endocrinol. Metab. 93: 2409-2412.

Fagin JA and Mitsiades N (2008). Molecular pathology of thyroid cancer: diagnostic and clinical implications. Best. Pract. Res. Clin. Endocrinol. Metab. 22: 955-969. 
Frates MC, Benson CB, Charboneau JW, Cibas ES, et al. (2006). Management of thyroid nodules detected at US: Society of Radiologists in Ultrasound consensus conference statement. Ultrasound Q 22: 231-238.

Gharib H, Papini E, Paschke R, Duick DS, et al. (2010). American Association of Clinical Endocrinologists, Associazione Medici Endocrinologi, and European Thyroid Association Medical guidelines for clinical practice for the diagnosis and management of thyroid nodules: executive summary of recommendations. Endocr. Pract. 16: 468-475.

Greene FL, Page DL, Fleming ID, Fritz AG, et al. (2002). AJCC Cancer Staging Manual. 6th edn. Springer-Verlag, New York.

Klinck GH and Winship T (1959). Psammoma bodies and thyroid cancer. Cancer 12: 656-662.

Lee ST, Kim SW, Ki CS, Jang JH, et al. (2012). Clinical implication of highly sensitive detection of the BRAF V600E mutation in fine-needle aspirations of thyroid nodules: a comparative analysis of three molecular assays in 4585 consecutive cases in a BRAF V600E mutation-prevalent area. J. Clin. Endocrinol. Metab. 97: 2299-2306.

Messina RL, Sanfilippo M, Vella V, Pandini G, et al. (2012). Reactivation of p53 mutants by prima-1 [corrected] in thyroid cancer cells. Int. J. Cancer 130: 2259-2270.

Miyakawa M (2012). Diagnostic imaging of thyroid tumor. Nihon Rinsho 70: 1900-1907.

Morris LF, Ragavendra N and Yeh MW (2008). Evidence-based assessment of the role of ultrasonography in the management of benign thyroid nodules. World J. Surg. 32: 1253-1263.

Nikiforov YE, Yip L and Nikiforova MN (2013). New strategies in diagnosing cancer in thyroid nodules: impact of molecular markers. Clin. Cancer Res. 19: 2283-2288.

Papini E, Guglielmi R, Bianchini A, Crescenzi A, et al. (2002). Risk of malignancy in nonpalpable thyroid nodules: predictive value of ultrasound and color-Doppler features. J. Clin. Endocrinol. Metab. 87: 1941-1946.

Qi HY, Zhao KJ, Ge LB, Hou YN, et al. (2012). Discussion for radial line characteristic of thyroid little nodules and the worth of qualitative diagnosis. Chin. J. Ctrl. Endem. Dis. 27: 71-74.

Rossi M, Buratto M, Bruni S, Filieri C, et al. (2012). Role of ultrasonographic/clinical profile, cytology, and BRAF V600E mutation evaluation in thyroid nodule screening for malignancy: a prospective study. J. Clin. Endocrinol. Metab. 97: 2354-2361.

Senchenkov A and Staren ED (2004). Ultrasound in head and neck surgery: thyroid, parathyroid, and cervical lymph nodes. Surg. Clin. North Am. 84: 973-1000. 